\title{
Lymphovenous Anastomoses Between Thoracic Duct and Azygos Vein in a Human Cadaver: A Case Report
}

\author{
Konstantinos N. Koutsouflianiotis ${ }^{1}$, George Paraskevas ${ }^{1}$, Maria Piagkou ${ }^{2}$, \\ George Noussios ${ }^{1}$, Konstantinos Natsis ${ }^{1}$
}

\author{
${ }^{1}$ Department of Anatomy and Surgical \\ Anatomy, Faculty of Health Sciences \\ School of Medicine, Aristotle University \\ of Thessaloniki, Thessaloniki, Greece \\ ${ }^{2}$ Department of Anatomy, School of \\ Medicine, Faculty of Health Sciences \\ National and Kapodistrian University \\ of Athens, Greece \\ Correspondence: \\ kostaskoutsouf@yahoo.gr \\ Tel.: + 302310952652 \\ Fax.: + 302310819831
}

Received: 1 February 2018

Accepted: 10 April 2018

Key words: Lymphovenous anastomoses Thoracic duct - Azygos vein.

\section{Introduction}

Lymphovenous anastomoses (LAs) in humans were described by Frantschi in 1948, who described communications between veins and lymphatics, especially at the height of the $12^{\text {th }}$ thoracic and the $2^{\text {nd }}$ lumbar vertebra, mostly connected with the system of the vena cava. Frantschi claimed that these anastomoses are of regular occurrence in humans too, since he had perfected his dissection technique and concluded that they exist in all cases, without exception (1). In contrast, post Frantschi researchers, such as Zhdanov (1952) and Griaznova (1953), strongly disagreed with the existence of the so-called "anastomoses of Frantschi" after meticulous dissection of a large number of human cadavers $(1,2)$. However, the presence of LAs has been proposed to protect the development of postmastectomy lymphedema and could also explain cases of rapid metastatic tumor evolution.

In the current study, LAs between the thoracic duct and the azygos vein are depicted and described, and a short review of the literature is provided in order for the reader to comprehend the anatomy, physiology, embryology and clinical applications of such communications.

\section{Case report}

During a routine dissection in our Department of Anatomy and Surgical Anatomy, we encountered LAs between the thoracic duct and the azygos venous system. The dis- 
section was conducted on an 80-year-old formalin-fixed male cadaver, used for educational and research purposes, whose death was unrelated to the present case report. Specifically, after meticulous dissection of the thorax region and the mediastinum, and after the excision of both lungs and the heart by means of the classical method of anatomical dissection, we detected LAs between the thoracic duct and the azygos vein. The specific cadaver displayed azygos, hemiazygos and accessory hemiazygos veins in the expected course in the mediastinum, whereas the thoracic duct was detected ascending normally through the posterior mediastinum between the azygos vein and the thoracic aorta. In particular, at the level of the fifth and sixth thoracic vertebra, two

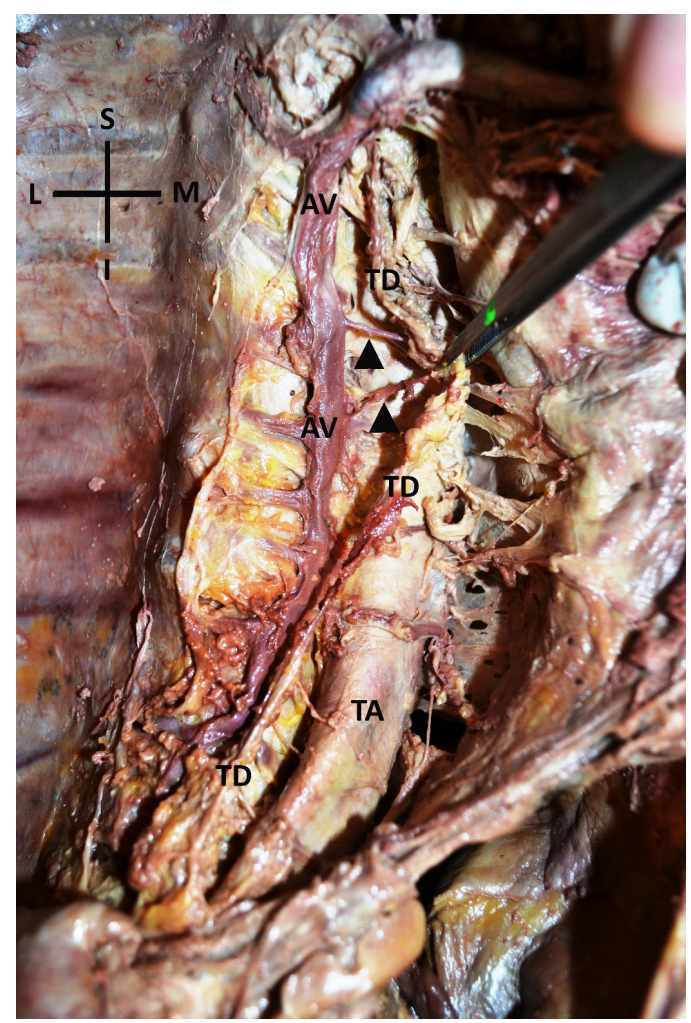

Figure 1. The azygos vein (AV) along with the thoracic duct (TD) in the posterior mediastinum are demonstrated communicating with two obliquely directed sizeable branches, the so-called lymphovenous anastomoses (arrowheads) (TA: thoracic aorta, S: superior, I: inferior, L: lateral, M: medial). sizeable veins arising from the azygos vein were directed obliquely to the trunk of the thoracic duct. The distance between the two LAs was $2.1 \mathrm{~cm}$, whereas the diameter of each LA was approximately $1.2 \mathrm{~mm}$ (Figure 1). Our findings were documented by several photographs taken using a Nikon D3100 digital camera, and the measurements were made using a digital vernier caliper with an accuracy of $0.01 \mathrm{~mm}$. No other congenital anomalies, variations or pathological conditions, or evidence of previous surgical interventions in the region were present.

\section{Discussion}

The origin of both azygos and hemiazygos veins is in the union of the ascending lumbar veins and the subcostal veins. The azygos vein ascends in the posterior mediastinum after it passes through the diaphragm, and terminates at the level of the fourth thoracic vertebra, arching anteriorly to the superior vena cava. The tributaries of the azygos vein are the posterior intercostal, bronchial, esophageal, pericardial and mediastinal veins (3). In contrast, both hemiazygos and accessory hemiazygos veins lie on the left side of the vertebral column in the mediastinum. Specifically, the first one begins, like the azygos, on the left, ascending to the eighth thoracic level, where it ends in the azygos vein. Tributaries of the hemiazygos are the lower three posterior intercostal veins, the esophageal and mediastinal branches, the left ascending lumbar vein, and the subcostal vein. The accessory hemiazygos vein receives blood from the fourth to eighth intercostals, the left bronchial veins, and often joins the hemiazygos and ends in the azygos vein (4).

The thoracic duct is a continuation of the cisterna chyli above the diaphragm, which lies between the abdominal aorta and the right crus of the diaphragm, and receives lymph from the abdomen and the 
lower limbs (5). The duct travels through the posterior mediastinum between the azygos vein and the thoracic aorta, and reaches the level of the sixth to fourth thoracic vertebra, where it crosses to the left side and extends to the root of the neck (6). It terminates at the junction of the left internal jugular vein and left subclavian vein at the base of the neck, receiving lymph from the left jugular and subclavian lymph trunks, in order to drain all the lymph of the body, apart from the right arm and the right halves of the thorax, head and neck (7).

The embryological origin of the azygos venous system could clarify the existence of such variations in the region, as in our study. The first network of lymphatic capillaries is dispensed along the primary, cardinal venous trunks. Six lymph sacs, which are paired, that is the jugular sacs, retroperitoneal sacs and posterior sacs, develop and consist of blood which is discharged into the nearby veins, thereby losing its vein communications. Down-growths from the jugular sacs unite with each other and meet upgrowths from the cisterna chyli, to produce the thoracic duct, which links the several sacs into a common system. The jugular sacs are the only ones to develop permanent connections with the vein system. Finally, the various sacs themselves break up into networks and are mainly replaced by chains of lymph glands (8). The abovementioned permanent connections between the jugular sacs and the veins could explain the LAs between the thoracic duct and the azygos vein, as in our case.

We consider that our reported case is exceptional since such a communication between the azygos vein and the thoracic duct is mentioned extremely rarely in the literature. To the best of our knowledge, such a case of a communication between the system of azygos veins and the thoracic duct was only mentioned recently by Dahran and Soames (9). In their study, these au- thors came across two branches of the thoracic duct, which drained into the accessory hemiazygos vein at the level of the eighth and ninth thoracic vertebra. Moreover, these tributaries were extremely small, less than 1 $\mathrm{mm}$ in diameter. In contrast, in our study the two LAs were between the thoracic duct and the azygos vein at the level of the fifth and sixth thoracic vertebra, while their size was approximately $1.2 \mathrm{~mm}$ in diameter. Van Limborgh observed these LAs in the thoracic prevertebral region in $50 \%$ of his cadaveric material, and concluded that these vessels are not true LAs, but constitute "vasa lymphatica vasorum" (10). Similarly, Griaznova, after perfusion of a gelatinous substance inside the thoracic duct in 10 human cadavers, was not able to detect any LA (2). It has been proposed that the normal presence of LAs in the upper limb may act as protection from postmastectomy lymphedema, since these vessels allow adequate lymph flow drainage from the arm, after radical excision of axillary lymph nodes (11). It has been shown that these LAs are more frequent in animals, and particularly in dogs. Specifically, de Freitas et al. observed such LAs between the thoracic duct and the azygous vein system in $60 \%$ of examined dogs, following ligature of the duct. However, under normal conditions, those authors failed to notice such LAs (12).

Thus, it may be suggested that these LAs could explain cases of rapid cancer spread from the lower part of the body, such as cancer of the gastrointestinal tract or pelvis, and lower limb tumors, to the lungs or other organs (9). Since the LAs in our study consist of sizeable vessels, it may be proposed that the hematogenous distribution of cancer cells from the lymph nodes to the arterial circulation is not only efficacious, but a short path is provided for rapid metastatic tumor evolution. 


\section{Conclusion}

Very few cases of LAs between the thoracic duct and the azygos vein have been recorded and published in the literature. Specifically, a double azygos-thoracic duct anastomosis is described in the current study, and could be an asset to present-day physicians to find an explanation and finally treat cases of rapid cancer spread. Moreover, the awareness of such LAs may be helpful for surgeons in the region when they come across vessels of an unknown nature, such as an anastomosis of the thoracic duct with the azygos vein.

\section{What is already known on this topic}

The existence of communications between the thoracic duct and the azygos vein has been a topic under discussion for many years, and they are considered to be extremely rare. Most researchers in the past, after dissecting a sufficient number of human cadavers, came to the conclusion that such communications do not exist in humans, but only in dogs.

\section{What this study adds}

This study adds important information regarding this very rare variation, that has been mentioned by very few researchers in the literature. A photograph of the case and an analytical description of the dissection are provided and a short review of the literature is given. The anastomosis between the thoracic duct and the azygos vein probably has embryological origin. Clinical considerations, explaining cases of rapid cancer spread, may arise for physicians.

Acknowledgments: We are grateful to Mrs Inga Iordanidou for her assistance during the translation of reference no 2 from the Russian language.

Authors' contributions: Conception and design: KK and GP; Acquisition, analysis and interpretation of data: KK, GP and MP; Drafting the article: KK, GP, GN and KN; Revising it critically for important intellectual content: KK, GP and GN; Approved final version of the manuscript: KK, GP, MP, GN and KN.
Conflict of interest: The authors declare that they have no conflict of interest.

\section{References}

1. Rusznyak I, Foldi M, Szabo G. Lymphatics and lymph circulation: physiology and pathology. 2nd ed. Oxford: Pergamon Press; 1967. p. 557.

2. Griaznova AV. Existence in man of lymphovenous anastomoses between the thoracic duct and the azygos and hemiazygos veins. Arkh Anat Gistol Embriol. 1953;30(2):40-4.

3. Rosse C, Gaddum-Rosse P. Hollinshead's Textbook of Anatomy. 5th ed. Philadelphia: Lippincott-Raven Publishers; 1997. p. 507-8.

4. Standring S, editor. Gray's Anatomy. 39th ed. Philadelphia: Elsevier Churchill Livingstone; 2008. p. 1026-7.

5. Ellis H. Clinical Anatomy. 11th ed. New Jersey: Blackwell Publishing; 2006. p. 45-7.

6. Bruce J, Walmsley R, Ross JA. Manual of surgical anatomy. London: E\&S Livingstone LTD; 1964. p. 152-3.

7. McMinn RMH. Last's Anatomy. 8th ed. Edinburgh: Churchill Livingstone; 1990. p. 281.

8. Arey LB. Developmental Anatomy. 6th ed. Philadelphia: W.B. Saunders Company; 1954. p. 390.

9. Dahran N, Soames R. Lymphovenous communications between the thoracic duct and the azygos venous system. Rev Arg de Anat Clin. 2015;7(1):60-3.

10. Van Limborgh J. On the nature of prevertebral lymphaticovenous communications of Frautschi (in French). Acta Anat. 1964;56:379-89.

11. Aboul-Enein A, Eshmawyl I, Arafa S, Abboud A. The role of lymphovenous communication in development of postmastectomy lymphedema. Surgery. 1984;95(5):562-6.

12. De Freitas V, Zorzetto NL, Prates JC, Seullner G. Experimental study of lymphatico-venous communications after thoracic duct ligature in dogs. Anat Anz. 1979;146(1):27-38. 